\title{
Subcutaneous Metastasis of Peritoneal Mesothelioma Diagnosed by Fine-Needle Aspiration
}

\author{
Lina PAPPA, ${ }^{1}$ Melpomeni MACHERA, ${ }^{1}$ Eleni TSANOU,${ }^{1}$ Constantina DAMALA, ${ }^{1}$ Dimitrios PESCHOS, ${ }^{2}$ \\ Maria BAFA, ${ }^{1}$ Vassiliki MALAMOU-MITSI ${ }^{1}$
}

${ }^{1}$ Department of Pathology-Cytology, ${ }^{2}$ Department of Forensic Medicine and Toxicology, University of Ioannina, Ioannina, Greece

\begin{abstract}
Mesothelioma is a rare malignant neoplasm of the serosal membranes, which can give distant metastases in various organs in advanced stages of its course. Subcutaneous tissue is an unusual metastatic site. In the literature, only one case of metastatic mesothelioma to the skin of the face has been reported. We present a case of a 60 -year-old female with a prior history of peritoneal malignant mesothelioma, who 6 months after the initial diagnosis presented with a subcutaneous nodule in the
\end{abstract}

lateral chest wall. Cytological examination of the material obtained by FNA from the nodule revealed metastatic mesothelioma. Although subcutaneous metastasis of malignant mesothelioma is a rare entity, one must always keep this possibility in mind and proceed to further investigation of such lesions. In these cases, FNA is a simple diagnostic procedure for the identification of metastatic disease in patients with a prior history of malignancy. (Pathology Oncology Research Vol 12, No 4, 247-250)

Key words: mesothelioma, metastatic nodules, FNA, cytology

\section{Introduction}

FNA is a simple, non-interventional and widely acceptable technique for the diagnosis of metastatic disease in subcutaneous nodules, especially in patients with an already known malignancy.

Malignant mesothelioma is a rare, invariably fatal neoplasm involving the serosal membranes predominantly of pleura and peritoneum in $95 \%$ of the cases. The ratio of these primary sites of origin is $2: 1{ }^{19,23}$ The neoplasm affects more men than women (2:1), ${ }^{4,17}$ Its incidence has increased during the last decades in the industrialized countries due to high asbestos exposure. ${ }^{2,12,29}$ Besides the occupational one, there is also an environmental etiology of the disease, which has been reported many years ago and concerns asbestiform fibers in the soil of several regions in European countries. ${ }^{6}$ One of these is the Metsovo area, which is located near the University Hospital of

Received: Febr 20, 2006; accepted: June 16, 2006

Correspondence: Vassiliki MALAMOU-MITSI, MD, PhD, Department of Pathology- Cytology, Medical School University of Ioannina, Ioannina 45110, Greece. Tel: +302651-0-97767, Fax: +302651-097893, E-mail: me00627@cc.uoi.gr or lina_pappa@yahoo.gr
Ioannina. Distant metastases may be observed in almost $50 \%$ of the cases. ${ }^{7}$ Most of them occur in the late stages of the disease, and usually they are discovered at autopsy. However, clinically evident distant metastases are rare, especially in the subcutaneous tissue.

The presented case is an example of this rare metastatic localization, in which the diagnosis was established by FNA cytology.

\section{Case Report}

A 60-year-old female was referred to the University Hospital of Ioannina, complaining of abdominal discomfort and pain. Ultrasound examination showed the presence of ascites and peritoneal thickening. The cytological examination of the aspirated ascitic fluid showed malignant mesothelioma of epithelioid type. The patient underwent laparotomy. The ovaries and fallopian tubes were resected along with a part of the peritoneum. The histologic examination revealed morphologic and immunohistochemical features of malignant mesothelioma. Six months after the initial diagnosis, during the scheduled follow-up and while the patient was still under chemotherapeutical treatment, a subcutaneous 


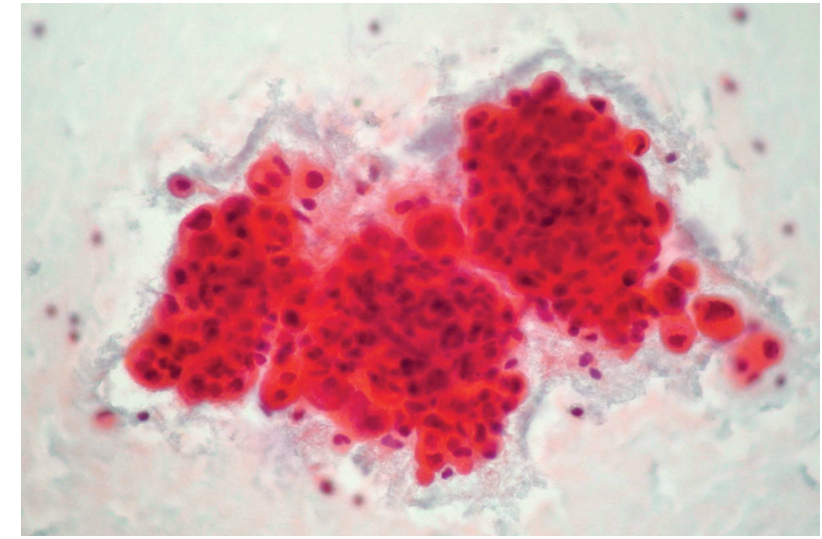

Figure 1. Ascitic fluid: atypical pleomorphic malignant cells of mesothelial origin, arranged in large papillary clusters with microvilli at the periphery and scattered lymphocytes and histiocytes in the background. (Papanicolaou, $x 40$ )

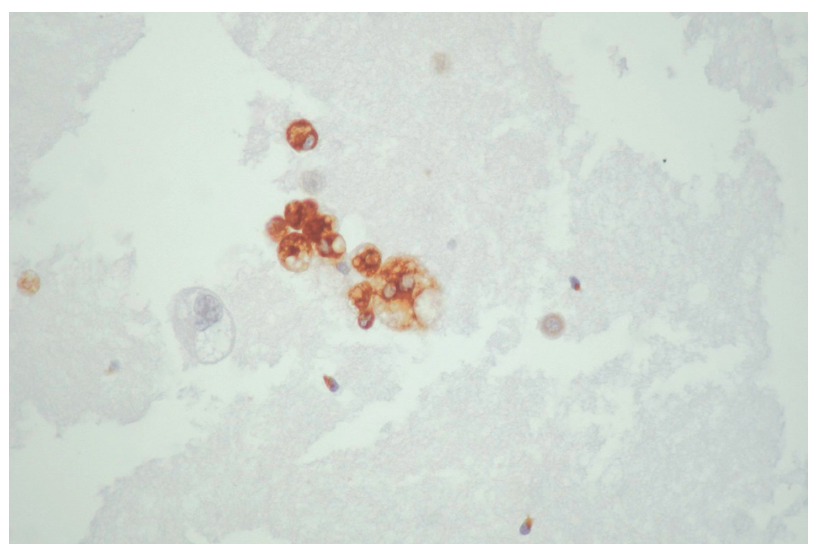

Figure 2. Immunopositivity of the neoplastic cells for vimentin in "cell block" preparation of the ascitic fluid (x40)

nodule of the lateral upper chest wall was discovered. CT scan was performed in order to rule out the possible existence of a synchronous pleural mesothelioma, and it was negative. An FNA biopsy of the nodule was performed and the cytological along with the immunocytochemical findings were consistent with metastatic malignant mesothelioma.

\section{Materials and Methods}

The ascitic fluid was sent from the Department of Internal Medicine and the FNA of the nodule was performed by a cytopathologist, using a 21-gauge needle attached to a 20 -ml syringe, which was mounted on a handle for singlehand grip. Three aspirations were obtained and the aspirated material was smeared on glass slides, alcohol-fixed or air-dried, and stained with the Papanicolaou and MayGrunwald-Giemsa technique, respectively. In order to confirm the diagnosis, the remaining material from the ascitic fluid was processed in "cell block" preparation, by centrifugation. The sediment was wrapped in filter paper, placed in a cassette, embedded in paraffin and $2-\mu \mathrm{m}$-thick sections were cut and stained immunocytochemically for carcinoembryonic antigen (CEA), epithelial membrane antigen (EMA), vimentin and Leu-M1.

For histologic examination of the resected peritoneum, tissue sections were fixed in $10 \%$ buffered formalin solution. Besides the morphologic evaluation on hematoxylin and eosin stained sections, immunohistochemistry was performed, applying the same panel of antibodies with the streptavidin avidin biotin (LSAB) method.

\section{Cytological findings}

The microscopic examination of the ascitic fluid revealed cellular smears containing many neoplastic cells with morphology of mesothelial lineage, in an inflammatory background, consisted mainly of lymphocytes. The neoplastic cells were arranged in clusters, usually with papillary configuration, sometimes provided with microvilli on their outer surfaces (Figure 1). The cytoplasm presented a perinuclear denser zone and the nuclei were irregular with prominent nucleoli. The described morphologic findings were suggestive of malignant mesothelioma. Immunocytochemically most of the malignant cells showed positivity for vimentin (Figure 2) and EMA, while CEA and Leu-M1 were negative. These results verified the diagnosis of malignant peritoneal mesothelioma.

The microscopic examination of the subcutaneous nodule showed cellular smears containing medium-sized atypical neoplastic cells, singly and in clusters or tissue fragments (Figure 3). The cells had pleomorphic atypical nuclei, rarely with intranuclear inclusions, and dense or vacuolated cytoplasm with evidence of necrosis (Fig-

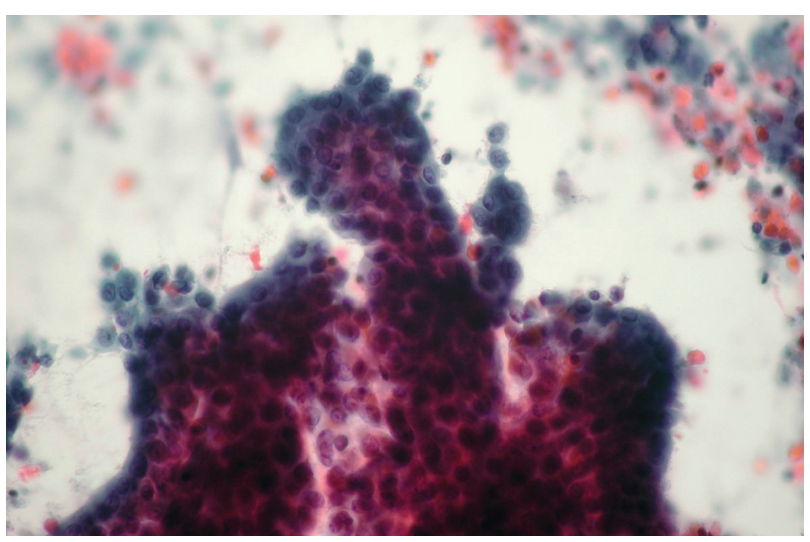

Figure 3. FNA smear of the subcutaneous nodule: papillary structures of atypical mesothelioma cells. Note the microvilli at the periphery of the cluster and the pleomorphic nuclei with nucleoli. (Papanicolaou, $x 40$ ) 


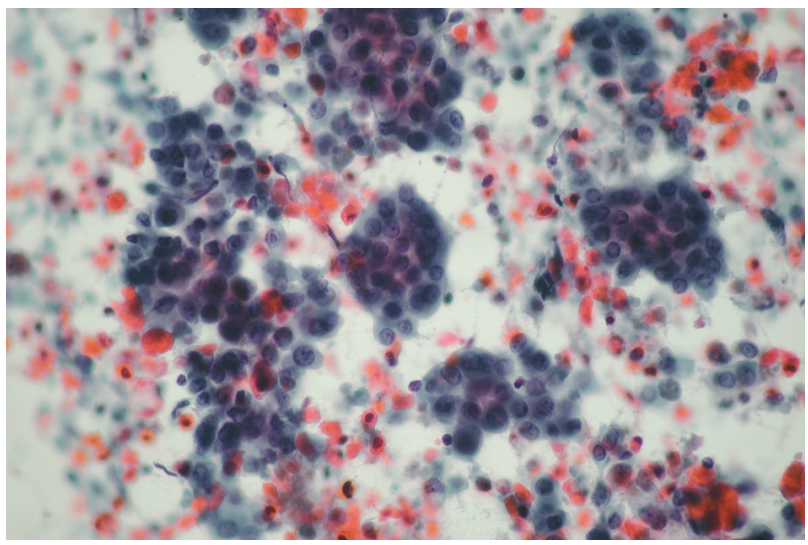

Figure 4. FNA smear of the subcutaneous nodule: increased cellularity with spheres of medium sized atypical neoplastic mesothelial cells in hemorrhagic and necrotic background. The cells have pleomorphic atypical nuclei and dense or vacuolated cytoplasm with evidence of necrosis (Papanicolaou, x40)

ure 4). The cytomorphological findings were consistent with metastasis of the previously diagnosed malignant peritoneal mesothelioma.

\section{Histological findings}

Histological examination of the resected peritoneum segment revealed invasion of the peritoneum from a malignant neoplasm with papillary and solid pattern of development. The tumor consisted of a monomorphous cell population with eosinophilic cytoplasm and moderate atypical nuclei with low mitotic activity (Figure 5). Immunohistochemical examination showed strong positivity for EMA and vimentin (Figure 6). Stains for cytokeratin, CEA and Leu-M1 were negative. These findings confirmed the cytological diagnosis of malignant mesothelioma. The ovaries and fallopian tubes had no pathologic findings.

\section{Discussion}

Malignant mesothelioma is a rare primary neoplasm affecting the serosal membranes with a relative increase of its incidence rate during the last decades, probably due to increased occupational exposure to asbestos. ${ }^{2,12,29}$ Furthermore, environmental exposure to asbestos, or asbestiform fibers (such as tremolite) is an already well-known entity manifested in the Metsovo area, a region near to our hospital, from which several cases of malignant pleural mesotheliomas have been reported. ${ }^{6,7}$ However, not all the cases of mesotheliomas are related to asbestos exposure, like in our case, in which such occupational or environmental risk factors ${ }^{5,18,19}$ were absent.

During its relative short course, this malignant neoplasm, independently of the therapy, can give local or distant hematogenous metastases in different organs. The reported metastatic sites include liver, lung, heart, brain, thyroid, adrenals, kidneys, pancreas, bone, soft tissue, skin and lymph nodes. It is important to mention that the majority of these metastatic foci are incidental autopsy findings. ${ }^{10,30}$

Only 16 previous cases of subcutaneous metastases of malignant mesothelioma have been reported. However, in 15 of the above reports the subcutaneous nodules concerned cases of pleural mesotheliomas and were located in the chest wall. Thus, these lesions were considered as local invasion of the disease. There is only one reported case of a pleural mesothelioma which gave rise to a distant subcutaneous metastasis in the face. ${ }^{9,10}$ Our case is the second report of a distant subcutaneous metastasis, and the first one diagnosed by FNA, since the former was diagnosed histologically by excisional biopsy.

Cytological evaluation of FNA aspirates from lesions clinically suspected for metastatic disease, such as enlarged lymph nodes and subcutaneous nodules, is usual-

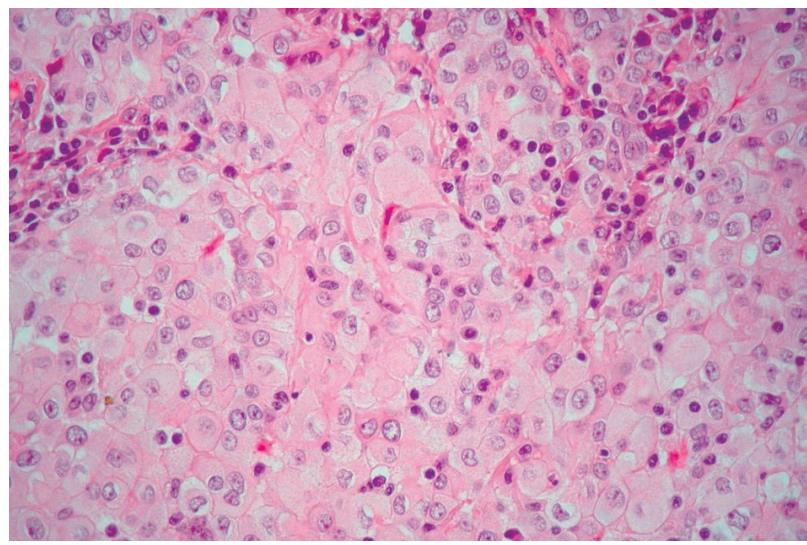

Figure 5. Histologic examination of the resected peritoneum: neoplastic monomorphous cell population, with eosinophilic or vacuolated cytoplasm and moderate atypical nuclei arranged in a solid pattern of development. (Hematoxylin $\mathcal{E}$ eosin, $x 40$ )

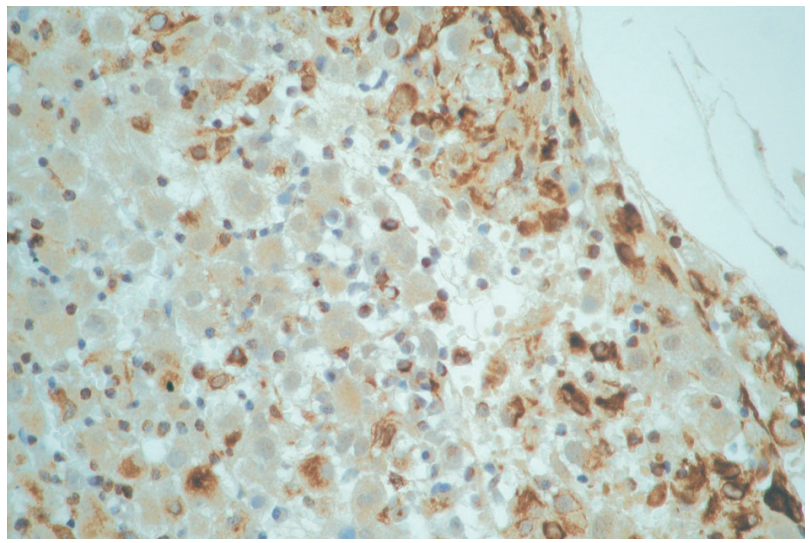

Figure 6. Immunopositivity for vimentin in tissue section of the resected peritoneum $(x 40)$ 
ly challenging. In case of a prior known history of malignancy, especially when there is archived material available for microscopic comparison, the diagnosis can be easily established with accuracy. ${ }^{1,3,20,27,32}$

The major differential diagnostic difficulty is the distinction between mesothelioma (of the tubulopapillary type) and adenocarcinoma. In these cases ancillary techniques such as immunocytochemistry can be helpful. Application of immunocytochemical stains reveals positivity for vimentin and EMA, while CEA and Leu-M1 are negative in cases of mesotheliomas. ${ }^{8,25,26}$ In our case the morphologic features along with the immunocytochemical findings were helpful for the establishment of the initial diagnosis. In the metastatic nodule the diagnosis was made, based only on the morphology and in comparison with the prior cyto-histological appearance.

In conclusion, FNA is a simple, cheap, non-invasive and accurate diagnostic procedure, therefore, it can be performed as a method of choice for the detection of occult metastatic lesions, especially in patients with an already known history of malignancy.

\section{References}

1. Ansari $N$, Derias $N$ : Diagnosis of malignant mesothelioma by fine needle aspiration of a cervical lymph node. A case report. Acta Cytol 44: 70-74, 2000

2. Antman $K$ : Natural history and epidemiology of malignant mesothelioma. Chest 103 (Suppl): 373-376, 1993

3. Battifora H, Kopinski M: Distinction of mesothelioma from adenocarcinoma: an immunohistochemical approach. Cancer 55: 1679-1685, 1985

4. Brenner J, Sordillo P, Magill G, Golbey R: Malignant mesothelioma of the pleura: Review of 123 patients. Cancer 49: 24312435, 1982

5. Chabot J, Beard D, Langlois A. Mesotheliomas of peritoneum epicardium and pericardium induced by strain of MC29 avian leucosis virus. Cancer Res 30: 1287-1308, 1970

6. Constantopoulos S, Goudevenos J, Saratzis N, et al: Pleura calcification and restrictive lung function in Northwestern Greece. Environ Res 38: 319-331, 1985

7. Constantopoulos S, Malamou-Mitsi V, Goudevenos J, et al: High incidence of malignant pleural mesothelioma in neighbouring villages of Northwestern Greece. Respiration 51: 266271,1987

8. Craig F, Fishback N, Scwartz J, Powers C: Occult metastatic mesothelioma - Diagnosis by fine needle aspiration. A case report. Am J Clin Pathol 97: 493-497, 1992

9. Dutt P, Baxter J, O'Malley F, et al: Distant cutaneous metastasis of pleural malignant mesothelioma. J Cutan Pathol 19: 490495, 1992

10. Edstrom L, Dawson P, Kohler J, DeMester T: Malignan mesothelioma. A metastasis to the face. J Surg Oncol 14: 251254, 1980

11. Garcia-Reija M, Matilla J, DePaz A, et al: Unusual metastasis to the mandibular alveolus of a malignant pleural mesothelioma. Otolaryngol Head Neck Surg 126: 435-437, 2002
12. Hillerdal G: Malignant mesothelioma 1982: Review of 4710 published cases. Br J Dis Chest 77: 321-343, 1983

13. Kaye J, Wang A: Malignant mesothelioma with brain metastases. Am J Med 80: 95-97, 1987

14. Kim B, Varkey B, Choi H: Diagnosis of malignant pleural mesothelioma by axillary lymph node biopsy. Chest 91: 278281, 1987

15. Kubota K, Furuse K, Kawahara M, et al: A case of malignant pleural mesothelioma with metastasis to the orbit. Jpn J Clin Oncol 26: 469-471, 1996

16. Losi L, Cocchi R, Calbucci F, Eusebi V: Metastasis of pleural malignant mesothelioma to the brain and upper maxilla: description of 2 cases. Pathologica 92: 273-277, 2000

17. MacDonald AD, Harper A, ElAttar O: Epidemiology of primary malignant mesothelial tumors in Canada. Cancer 26: 914 919,1970

18. McEwen J, Finlayson A, Mair A: Mesothelioma in Scotland. Br Med J 4: 575-578, 1970

19. Moertel CG: Peritoneal mesothelioma. Gastroenterology 63: 346-350, 1972

20. Obers V, Leiman G, Girdwood R, Spiro F: Primary malignan pleural tumors (mesotheliomas) presenting as localized masses. Fine needle aspiration cytologic findings, clinical and radiologic features and review of the literature. Acta Cytol 32: 568-575, 1988

21. Prieto $V$, Kenet $B$, Varghese $M$ : Malignant mesothelioma metastatic to the skin presenting as inflammatory carcinoma. Am J Dermatopathol 19: 261-265, 1997

22. Roberts G: Distant visceral metastases in pleural mesothelioma. Br J Dis Chest 70: 246-250, 1976

23. Sebbag $G$, Sugarbaker P: Peritoneal mesothelioma proposal for a staging system. Eur J Surg Oncol 27: 223-224, 2001

24. Sgrignoli A, Abati A, Pass H, et al: Metastatic mesothelioma presenting as a salivary gland neoplasm. Acta Cytol 42: 818820,1998

25. Sheibani K, Esteban J, Bailey A, et al: Immunopathologic and molecular studies as an aid to the diagnosis of malignant mesothelioma. Hum Pathol 23: 107-116, 1992

26. Sheibani K, Shinn S, Kezirian J, Weiss L: BerEP4 antibody as a discriminant in the differential diagnosis of malignant mesothelioma versus adenocarcinoma. Am J Surg Pathol 15: 79-84, 1991

27. Sterret $G$, Whitaker $D$, Shilkin $K$, Walters $M N$ : Fine needle aspiration cytology of malignant mesothelioma. Acta Cytol 31: $185-193,1987$

28. Sussman J, Rosai J: Lymph node metastasis as the initial manifestation of malignant mesothelioma. Report of six cases. Am J Surg Pathol 14: 819-828, 1990

29. Wagner J, Sleggs C, Marshad P: Diffuse pleural mesothelioma and asbestos exposure in the North Western Cape Province. $\mathrm{Br}$ J Ind Med 17: 260-271, 1960

30. Walters $K$, Martinez A: Malignant fibrous mesothelioma metastatic to brain and liver. Acta Neuropathol 33: 173-177, 1975

31. Wanebo H, Martini N, Melamed M, et al: Pleural mesothelioma. Cancer 38: 2481-2488, 1976

32. Yu G, Baloch Z, Gupta P: Cytomorphology of metastatic mesothelioma in fine needle aspiration specimens. Diagn Cytopathol 20: 328-332, 1999

33. Zanconati F, DelConte A, Bonifacio-Gori D, Falconieri G. Metastatic pleural mesothelioma presenting with solitary involvement of the tongue: a report of a new case and review of the literature. Int J Surg Pathol 11: 51-55, 2003 